\title{
La necesidad de un Código de Construcción Sostenible para Bogotá: ¿Cómo construir una propuesta?1
}

\author{
The Need for a Sustainable Building Code for Bogotá: Creating a Proposal
}

\author{
Clemencia Escallón ${ }^{(1)}$, Camilo Villate ${ }^{(2)}$ \\ (1) Magíster en Estudios Interdisciplinarios sobre Desarrollo. Profesora de la Facultad de Arquitectura de la Universidad de los Andes. \\ Bogotá, Colombia. cescallo@uniandes.edu.co \\ (2) Magíster en Tecnologías de la Construcción de la Universidad Nacional de Colombia. Profesor de la Facultad de Arquitectura de la \\ Universidad de los Andes. Bogotá, Colombia. cvillate@uniandes.edu.co
}

Recibido 8 de septiembre de 2014. Modificado 13 de febrero de 2015. Aprobado 17 de febrero de 2015.

DOI: http://dx.doi.org/10.16924/riua.v0i41.775

\begin{abstract}
Palabras clave
Bogotá, código, estándar, marco conceptual, sostenibilidad, Universidad de los Andes.

Resumen

La Universidad de los Andes recibió en 2010 el encargo, desde las secretarías de Planeación y Ambiente del Distrito Capital, de revisar, evaluar y diagnosticar el actual Código de Construcción de Bogotá y proponer el marco conceptual que permitiera su actualización a la luz de la sostenibilidad. En este artículo se comentarán las diferencias entre código y estándar, el enfoque planteado para la revisión y las estrategias propuestas por el equipo de la Universidad de los Andes.
\end{abstract}

\section{Key words}

Bogota, code, conceptual framework, standard, sustainability, Universidad de los Andes.

\begin{abstract}
In 2010, the Planning and Environment Secretaries of the Capital District asked Universidad de los Andes to review, evaluate and assess the current building code in Bogota, and put forward a conceptual framework that would allow the code to be updated with respect to sustainability. This article describes the differences between the code and the standard, the approach used for the review, and the strategies proposed by the team from Universidad de los Andes.
\end{abstract}

\section{INTRODUCCIÓN}

Hace tres años, varias dependencias de la Universidad de los Andes - el Departamento de Ingeniería Civil y Ambiental, el Departamento de Arquitectura y la Facultad de Derechoparticiparon en la revisión y propuesta de actualización del código de construcción de Bogotá.

En 2010 se solicitó a la Universidad acompañar la solicitud del Concejo de la ciudad para la revisión del Código de Construcción vigente bajo el enfoque de sostenibilidad. Lo primero que hizo el grupo de la Universidad fue revisar qué estaba pasando en el contexto mundial, cómo se regulaba en otras latitudes el ejercicio de la construcción y se puso mucho cuidado en entender los códigos, los estándares y las guías. Como se muestra en la Figura 1, desde un nivel ascendente de obligatoriedad, desde un código que busca como norma regular un tema específico, en este caso el de la construcción, seguido por los estándares como pautas que se dan para permitir nuevos escenarios, modelos y procesos; y las guías de buenas prácticas en las que se demuestran avances y posibilidades. A partir de este ejercicio, quedó claro que se estaba en un escenario de código de construcción, en un ámbito de obligatoriedad.

En esta oportunidad se revisaron diecisiete documentos entre códigos y estándares en distintas partes del mundo: Norteamérica, la región de América Latina, Europa y Oceanía para entender

${ }^{1}$ Este artículo es el resultado de la ponencia presentada por Clemencia Escallón y Camilo Villate en el foro "Los códigos en la construcción en Colombia: Aproximaciones y Aplicaciones", realizado en la Universidad de los Andes el 8 de septiembre de 2014. 
bien cómo estaban estructurados los documentos, qué temas y perspectivas abordaban, cómo se manejaba a los actores, qué retos se planteaban y cómo se aproximaban en términos de la sociedad, la tecnología y el mismo territorio en el que se insertaban.

Uno de los aspectos que más interesó fue entender la relación entre código y estándar y la responsabilidad que se tiene en esa relación (Figura 2). Los mínimos que se regulan en un código y que son obligatorios deben ser entendidos colectivamente en un proceso que debe involucrar a todos los actores. Además de ser factibles y compartidos en una sociedad, los mínimos deben cubrir el máximo de elementos dentro de esa actividad edificadora. En el caso de Bogotá, por ejemplo, en el diagnóstico se evidenció que el $70 \%$ de las construcciones eran vivienda, por lo que un código de construcción debía atender de manera prioritaria este componente urbano. Por otra parte, un altísimo porcentaje de la actividad edificadora está fuera de la norma, puesto que se construye en escenarios de ilegalidad e informalidad, así que el código también debía tener en cuenta esta condición especial. Estos aspectos propios del caso de Bogotá había que tenerlos en cuenta en la revisión del código.

\section{¿Cómo fue El CONVENio? ¿QUÉ ESTABA PASANDO EN Bogotá?}

La Universidad de los Andes inició su participación en el Código de Construcción de Bogotá en 1985, cuando desde las entidades del Distrito la convocaron para elaborar el primer documento que finalmente fue aprobado por el Concejo Distrital, sin modificaciones, bajo el Acuerdo 20 de 1995. Esta situación generó varios problemas: por un lado, al no actualizarse reflejaba el estado del arte de la década anterior y por otro, no incorporaba cambios importantes en las normas de sismo resistencia, ni cambios en el esquema y procedimiento de licenciamiento desde las curadurías; ni incorporaba normas

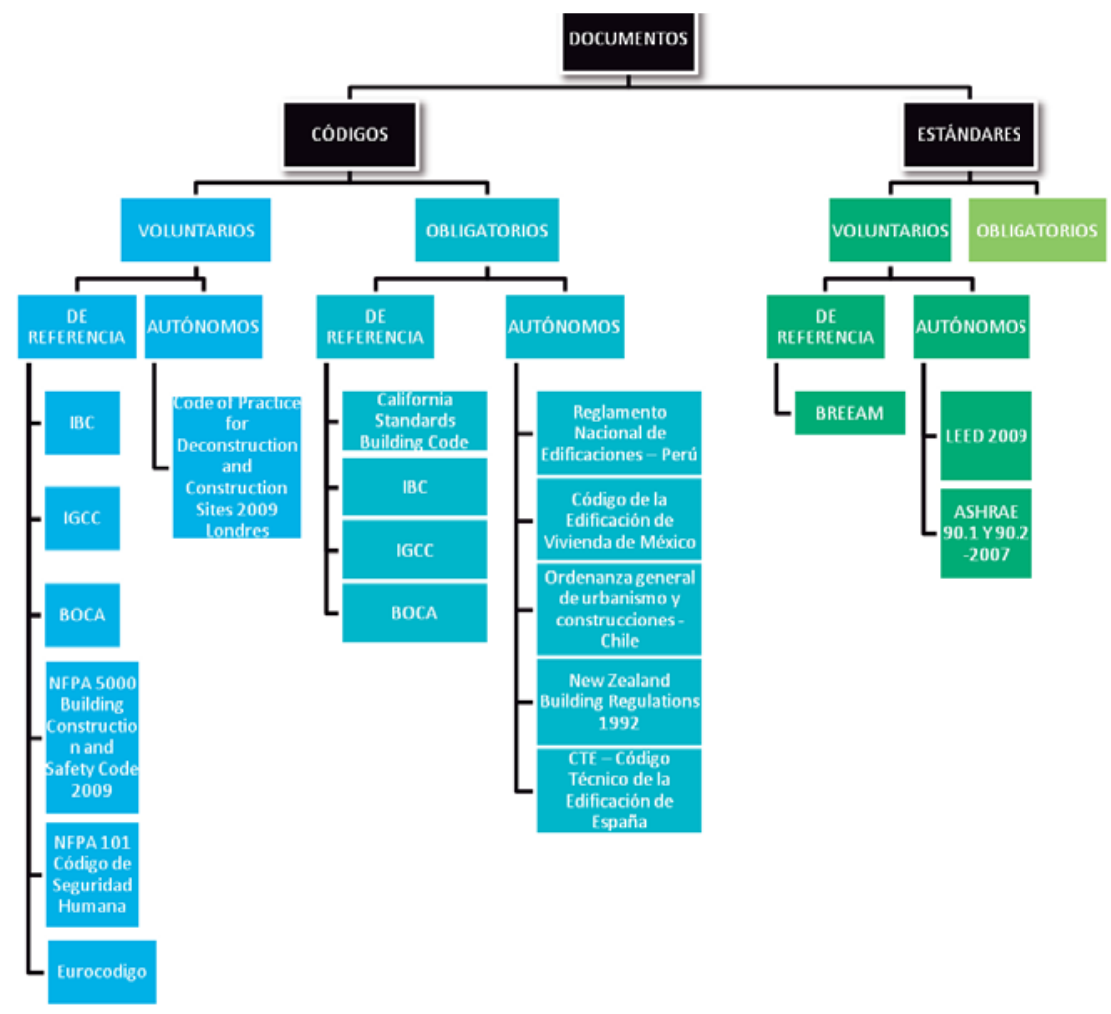

Figura 1. Código y estándar

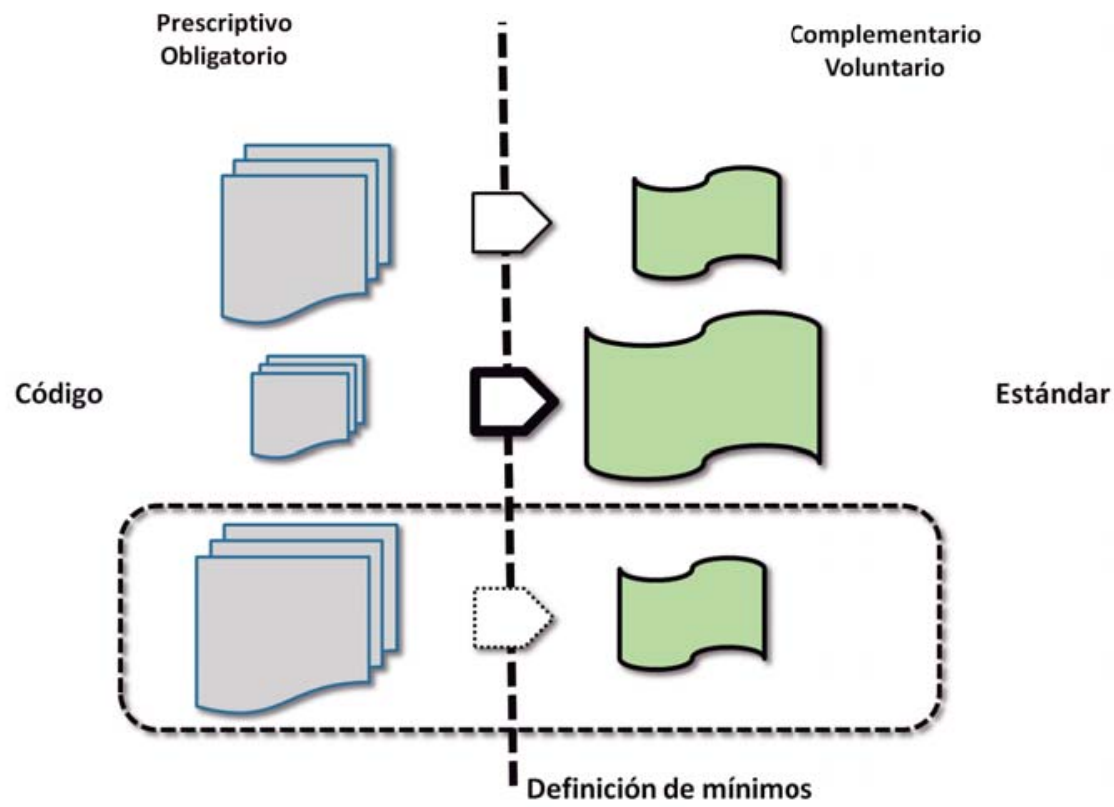

Figura 2. Código y estándar

recientes en relación con servicios públicos. Todo lo anterior generó un escenario de bajísima legitimidad que afectó de manera importante su aplicación entre los actores involucrados, particularmente, los constructores.
En 2008, el Concejo de la ciudad le pidió a la Administración que hiciera una actualización del código e incorporara el enfoque de sostenibilidad, solicitud que motivó la convocatoria que hicieron las Secretarías Distritales y en 
la que la Universidad de los Andes participó para evaluar la pertinencia técnica de las normas del código vigente y crear una propuesta de actualización. Para el desarrollo de las actividades se firmó un convenio y se conformó un equipo de trabajo con las distintas áreas académicas que participaron en el proyecto.

\section{Características del Código de CONSTRuCCión de Bogotá}

El código de construcción vigente para Bogotá es un código de tipo prescriptivo y se apoya en escenarios y condiciones de los años ochenta. Cuando se hizo la revisión conjunta con los funcionarios de la Administración, se evidenció que la pertinencia técnica era muy alta y que un $90 \%$ de los temas mantenían aún su vigencia y se identificaron los nuevos escenarios normativos que debían formar parte de la revisión, por ejemplo, en el diseño arquitectónico, instalaciones, licencias, consideraciones de construcción y de ocupación.

Ahora bien, la cantidad de normas que se vinculan a la regulación de la construcción son, en el caso de Bogotá, múltiples y de alta complejidad. En el análisis adelantado se identificaron 660 normas que debe tener en cuenta un curador en el desempeño de su función y que deben estar vinculadas temáticamente al código de construcción.

La Figura 3 titulada "Somos parte del proceso” elaborada para alguna reunión de trabajo, sirve para comprender el enfoque de la revisión. Es un proceso donde lo principal es entender el territorio y los impactos que tiene la edificación y donde además es necesario reconocer colectivamente los parámetros para medir ese impacto. Al estar vinculados los actores en el reconocimiento del proceso y prestar atención a la actualización del código, es posible mantener su vigencia y generar un sentido como herramienta para todos los actores. Lo anterior, con la convicción de que un código debe ser una construcción colectiva, es decir, debe tener sentido para los distintos actores en todos los momentos en los que se requiera su aplicación.

Como equipo de profesionales, desde la Universidad era prioritario mantener

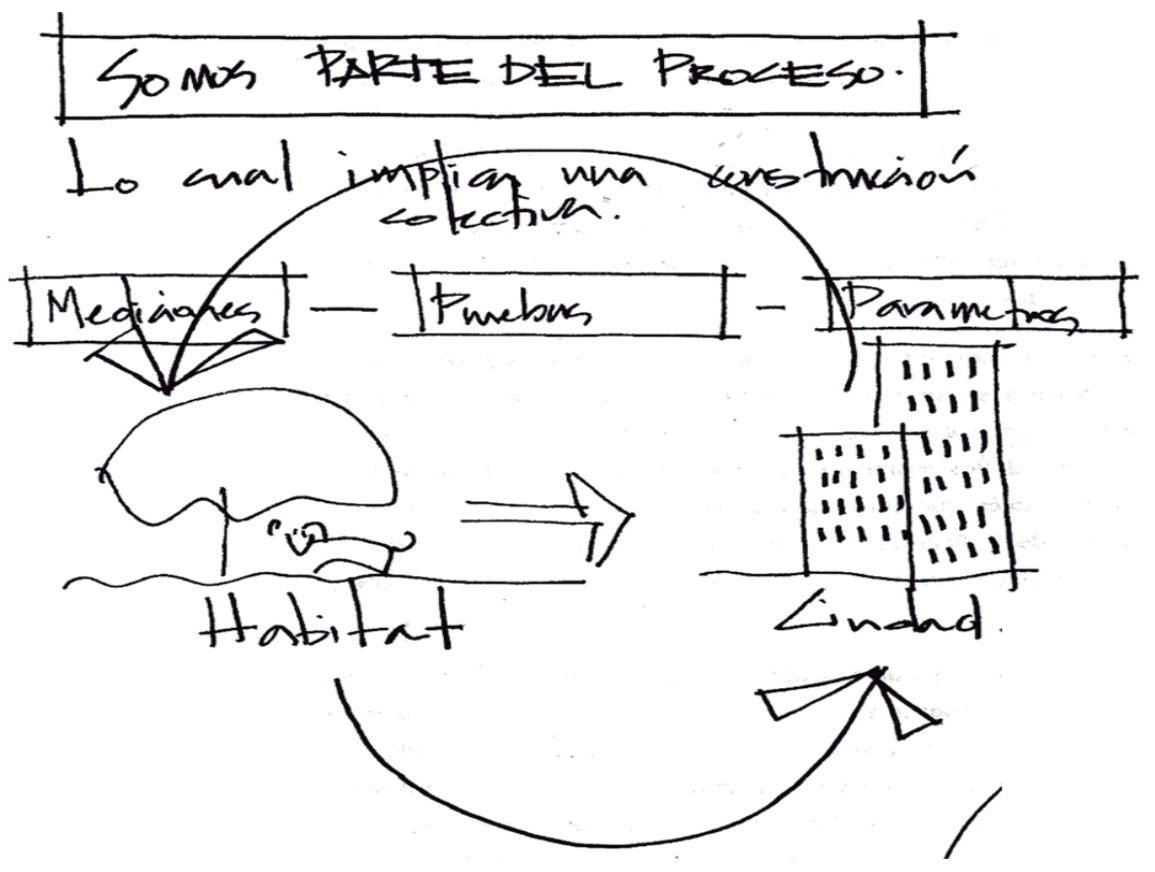

Figura 3. "Somos parte del proceso"

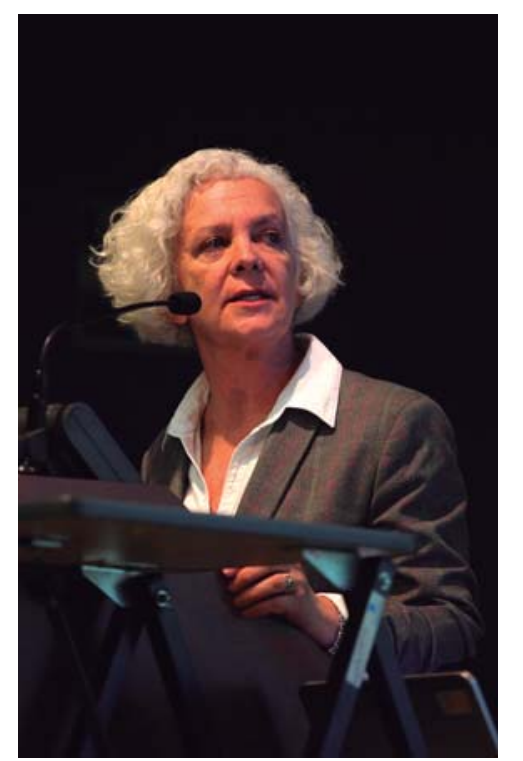

el enfoque de sostenibilidad para la propuesta de actualización del código. En esa búsqueda se mantuvieron presentes tres conceptos principales: seguridad, salubridad y bienestar de la comunidad, con las dificultades propias de abordar desde allí el nuevo enfoque de sostenibilidad.

\section{¿Cómo Se Construye un CÓDIGo?}

Había que pensar en la construcción del código no solo desde sus conceptos sino desde su operación y logística. En este sentido, fue fundamental apoyarse para la reflexión en imágenes de los problemas de la ciudad como los que se observan en la Foto 1. Esto es un reflejo de cómo construimos en Bogotá, es decir, por cada nuevo metro cuadrado de construcción, se generan alrededor de 1,6 a 1,8 metros cúbicos de escombros que pueden terminar en humedales. Esto es gravísimo y con ello lo importante es resaltar la necesidad en Bogotá de regular y organizar la actividad de la construcción en general.

En una visión compleja multidimensional de muchas variables que intervienen en el proceso de construcción, en esta oportunidad se propuso al Distrito una estrategia para organizar el código. Como se muestra en la Figura 4, es un proceso que inicia con el Plan de Ordenamiento Territorial 
(POT) desde donde se busca el equilibrio en la ocupación del territorio. En el caso de Bogotá, el POT vigente se fundamenta de manera adecuada en la estructura ecológica principal. Tomando ese punto como la estrategia para ocupar el territorio, se debería conectar con una escala intermedia, es decir, un código de urbanismo, para finalmente inscribirse en el ámbito propio de la aplicación del código de construcción, del paramento hacia adentro, y hablar de sostenibilidad.

\section{¿Cómo hablar de SOSTENIBILIDAD EN UN CÓDIGO DE CONSTRUCCIÓN?}

El acuerdo del grupo de trabajo en la revisión del código fue partir de un autor, Jason McLennan, quien propone principios de sostenibilidad que se aplicaron en acciones que promueven fundamentalmente respeto por los semejantes, el medio ambiente y los materiales, entre otros. Fue importante hablar, por ejemplo, de temas de seguridad de la vida y lograr identificar el respeto por los semejantes como un tema de sostenibilidad y desde allí tener en cuenta los temas de seguridad. Esa reflexión se cruzó con el ciclo de vida de la edificación en las etapas principales. Cualquier visión de sostenibilidad no puede desconocer la importancia de entender los objetos construidos a partir del ciclo de vida, inclusive cerrando el ciclo con la disposición final donde se recuperan elementos y materiales que vuelven a entrar al proceso.

Las áreas temáticas que tiene el documento técnico del código de construcción de 1985 son territorio, arquitectura, construcción, estructuras, instalaciones, seguridad y energía, y no se cambiaron. Se cruzaron con la visión de los diferentes actores que intervenían en el código, dejando claro que no era solamente tema de arquitectos e ingenieros sino de todo aquel que tenía que ver con la construcción - el administrador del edificio quien lo recibe en sus zonas comunes y debe mantenerlo operando adecuadamente como esta

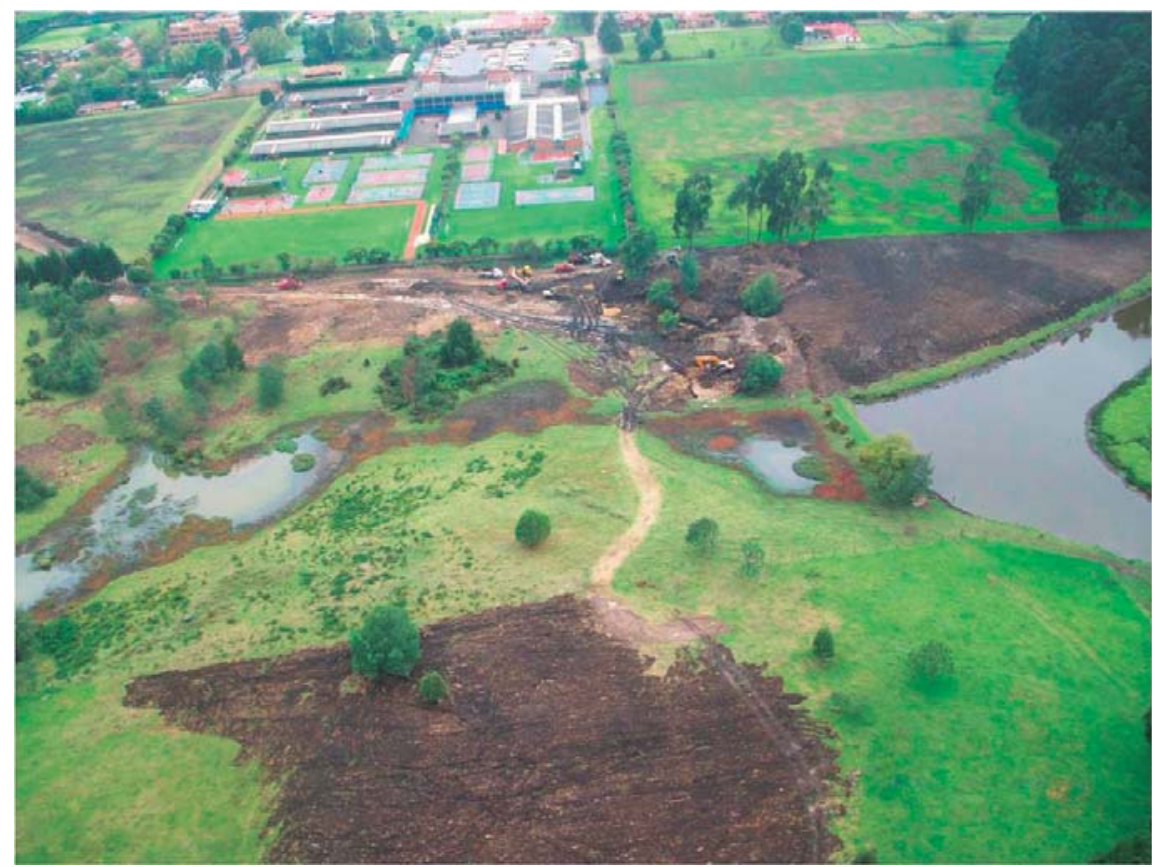

Foto 1.

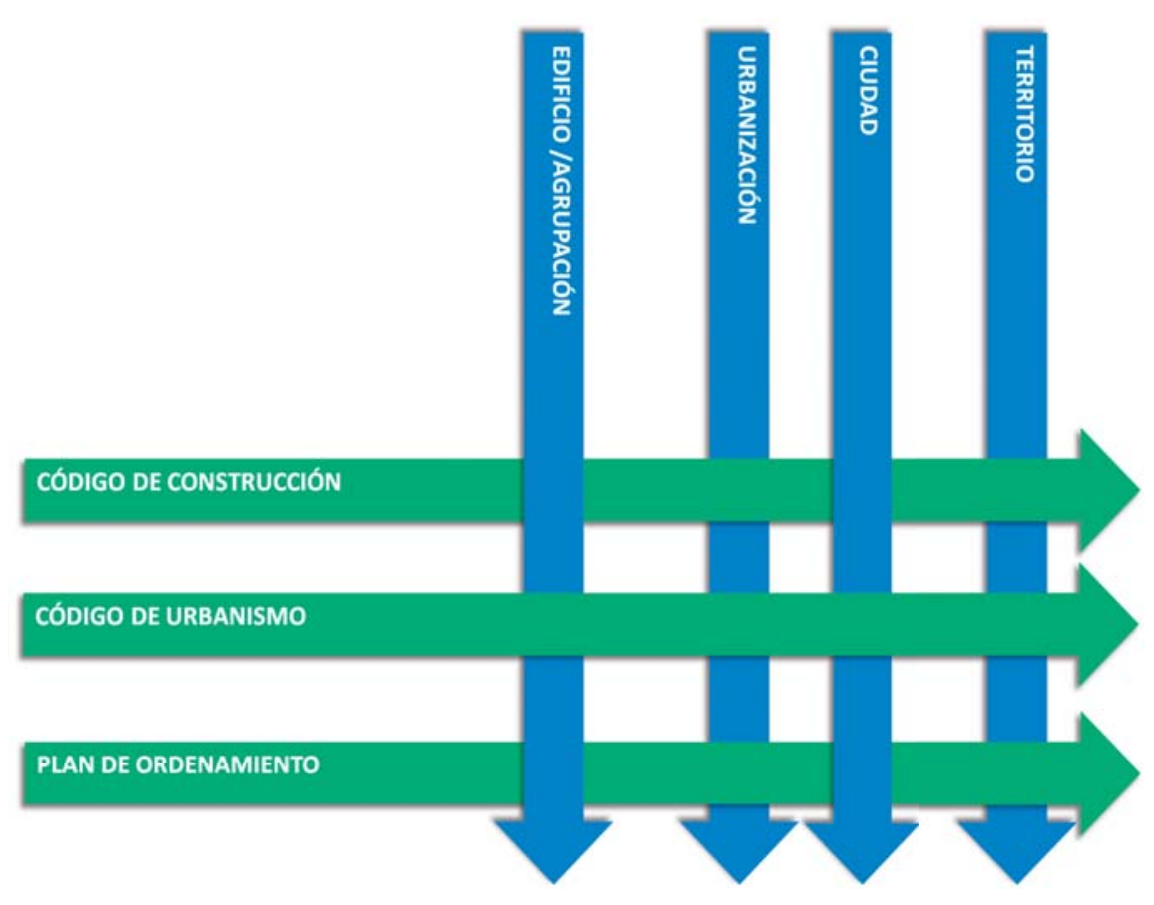

Figura 4. (POT)

planeado; el Distrito desde todas las entidades distritales; hasta el mismo sector bancario- - En fin, todos los actores fundamentales del proceso para que ese código que se necesita reformular y actualizar se constituya en un instrumento legítimo. Fue muy importante en su momento entender bien cuál era el papel de la Comisión de Revisión del Código de Construcción Sostenible de Bogotá, y a quien le correspondía también preocuparse por mantener el 
Código actualizado, vigente y legítimo como se planteaba.

\section{MATRIZ MULTIPROPÓSITO}

La elaboración de una matriz multipropósito pasó por relacionar los temas del código de construcción con el ciclo de vida de la edificación y los propósitos de sostenibilidad, para identificar y, en el cruce de ellos, definir el tipo de acciones a seguir. El encargo principal fue definir la estructura conceptual para ese código de construcción. Se identificó la oportunidad para vincular la actividad de la edificación en Bogotá, es decir, tener una herramienta que permitiera, dependiendo del papel dentro del proceso de la construcción, entrar de diferente manera e identificar las acciones específicas para hablar de construcción adecuada y sostenible en la ciudad.

El Código de Construcción Sostenible se pensó como un gran contenedor de múltiples acciones que desde diferentes entradas, dependiendo del papel, —administrador de un edificio, diseñador arquitectónico, diseñador estructural-, permitiera encontrar los lineamientos a tener en cuenta de una manera clara, como un manual de operación, para aplicar en la construcción en Bogotá (Figura 5). La información se detalló en múltiples fichas que como acciones específicas, en su conjunto, se convierten en el código de construcción.

Se buscaba con el Código de Construcción de Bogotá, que hoy está parcialmente vigente desde lo técnico y desde lo jurídico, que en el proceso de socialización de su actualización se convirtiera en una herramienta con mucha legitimidad. La estrategia para su implementación y actualización es fundamental. Se consideró también que era la oportunidad que tenía el Distrito y la ciudad para unificar y alinear el código con las reglas de carácter nacional y local de mayor jerarquía, por ejemplo, la norma sismo resistente.

Como conclusión se puede decir que la tarea está por hacerse. Entendemos que el Distrito hoy está adelantando

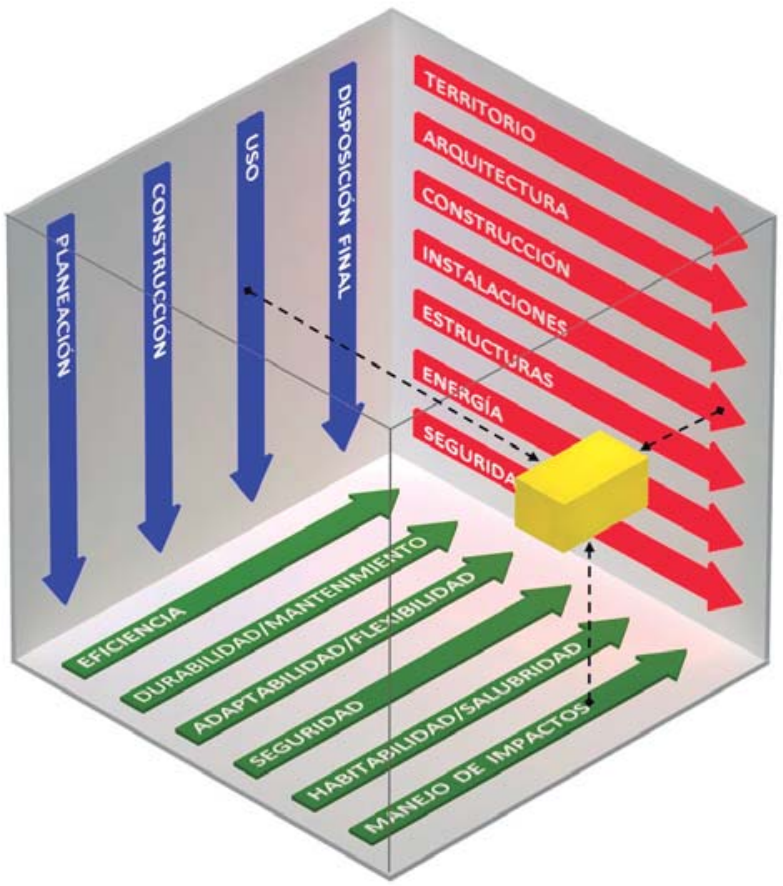

Figura 5. Matriz multipropósito

acciones cuando menciona la política de construcción sostenible y el ecourbanismo, temas importantes que pretende promover la Secretaría de Ambiente, pero que se puede traducir en ejercicios aislados que deberían estar vinculados a la revisión del Código de Construcción. Es la oportunidad para que, efectivamente, la vivienda entre en un proceso de edificación sostenible y no solamente las oficinas, que

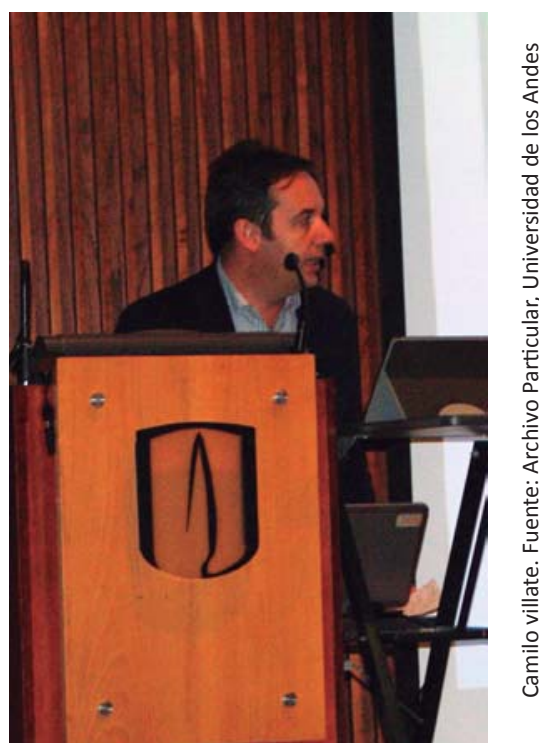

son un porcentaje menor, y que si bien son certificadas con estándares LEED, realmente no transforman la situación actual de la ciudad.

\section{REFERENCIAS}

Concejo de Bogotá. (1995). Acuerdo 20 de 1995, por el cual se adopta el Código de Construcción del Distrito Capital de Bogotá, se fijan sus políticas generales y su alcance, se establecen los mecanismos para su aplicación, se fijan plazos para su reglamentación prioritaria y se señalan mecanismos para su actualización y vigilancia. Bogotá, Colombia.

Concejo de Bogotá. (2008). Acuerdo 323 de 2008, por el cual se le otorga un plazo de (2) años al Alcalde Mayor para incorporar en el Código de Construcción de Bogotá, el Estándar Único de Construcciones Sostenibles para el Distrito Capital y de ajustar dicho Código al estándar en mención.

McLennan, J. F. (2004). The Philosophy Of Sustainable Design. Kansas City: Ecotone LLC. 